\title{
Regulatory network of microRNAs and genes in testicular cancer
}

\author{
YANSONG ZHAO ${ }^{1,2}$, ZHIWEN XU $^{1,2^{*}}$, NING WANG ${ }^{1,2}$ and SHANG WANG ${ }^{1,2}$ \\ ${ }^{1}$ Key Laboratory of Symbol Computation and Knowledge Engineering of The Ministry of Education; \\ ${ }^{2}$ Department of Computer Science and Technology, Jilin University, Changchun, Jilin 130012, P.R. China
}

Received March 20, 2015; Accepted April 12, 2016

DOI: $10.3892 / 01.2016 .5043$

\begin{abstract}
Testicular cancer (TC) is the most common cancer in men between 20-40 years of age. A large number of studies have focused on identifying the cause of this disease; however, the underlying regulatory mechanisms have not been thoroughly investigated and the specific cause remains unclear. The present study systematically analyzed the regulatory associations between genes, transcription factors (TFs) and microRNAs (miRNAs), aiming to obtain key information regarding the regulatory processes of TC. Three different networks were derived from the analysis: Global, related and differentially-expressed. These networks may be able to identify the primary causes of TC through gene analysis, which determines underlying regulatory pathways and subsequently discloses information regarding TC pathology. The differentially-expressed network is considered to be the most important. If the differentially-expressed elements in this network were to be manipulated back to normal levels via human intervention, this may prevent the onset of TC. This may be described as suppressing TC at the genetic level. If the abnormal expression of these elements was to be corrected, then preventing TC at the source may be a feasible option. Thus, the present study compared and analyzed the global, related and differentially-expressed networks, from which important genetic pathways in TC were highlighted. In addition, self-adaptation associations, host genes and target genes were analyzed. The upstream and downstream elements were identified, and TFs were predicted using the P-match method. When combined, the results of the current study provide the
\end{abstract}

Correspondence to: Miss. Shang Wang, Department of Computer Science and Technology, Jilin University, 2699 Qianjin Street, Changchun, Jilin 130012, P.R. China

E-mail: wangshang@jlu.edu.cn

\section{*Deceased}

Abbreviations: miRNA, microRNA; TFs, transcription factors; NCBI, national center for biotechnology information; TC, testicular cancer

Key words: testicular cancer, network, transcription factor, microRNA, host gene, regulatory pathway basic materials for further research on important genes in TC, and provide guidance on the pathological curative method.

\section{Introduction}

Testicular cancer (TC) is a frequently occurring disease among adult males (1). TC develops in the testicles and may include various types of cancer, including germ cell tumors (GCT), sex cord or gonadal stromal tumors, and secondary tumors of the testes (1). Over $95 \%$ of TC cases are testicular GCTs (TGCTs) (2). Although TC has the highest cure rate of all forms of cancer and a 5-year survival rate of $>90 \%$ (1), TC remains to be the most common cancer in young to middle-aged men (3).

MicroRNAs (miRNAs/miRs) and transcription factors (TFs) are key regulators of gene expression (4). TFs are specific proteins that control transcription by regulating gene expression at the transcriptional level, either working alone or with other proteins. TFs function to activate or repress transcription by binding to specific DNA sequences, known as cis-regulatory elements, located in the upstream regions of genes, whilst miRNAs are small, non-coding RNA molecules that affect gene expression at the post-transcription level (4).

miRNAs target genes that are associated with, and are important in, a number of biological processes, including apoptosis, differentiation and proliferation. Studying these target genes (targets) offers an intuitive method to identify the effect of miRNAs at the transcriptional level. Genes that contain miRNAs are known as host genes (hosts). Rodriguez et al (5) reported that miRNAs and host transcripts are transcribed in parallel, and identified two transcription classes of miRNAs, known as intronic and exonic. Baskerville and Bartel (6) demonstrated that there is a close association between host genes and their intronic miRNA, which are often expressed together, forming a specific association that allows them to achieve full biological function and subsequently modify pathways (7).

miRNAs and differentially-expressed genes involved in TC have been investigated for $>10$ years in this century. However, studies at the gene level are currently only based on a single aspect, either genes or miRNAs. As a result, identifying the genes of interest in the pathogenesis of TC is challenging. The present study aimed to take a more global perspective, determining the underlying networks of miRNAs, TFs, targets and hosts to analyze the basic gene pathogenesis. The results identified three types of association: miRNAs target genes, and TFs regulate miRNAs and miRNA host genes. The global network is necessary for research, but cannot be 
used easily to extract the key regulatory elements; therefore, differentially-expressed and related networks were also determined. The latter two networks were used specifically to extract key pathways sustained by differentially-expressed elements. To compare the similarities and differences between these pathways, the current study identified key elements at the gene level of TC regulation. The results of the present study reveal the regulatory associations of TC and are important in further aiding the research in this field. If mutated factors can be regulated and maintained within normal ranges, the development of TC may be prevented.

\section{Materials and methods}

Material collection and data processing. Firstly, the present study collected experimentally validated datasets of human TC miRNAs and their target genes from Tarbase 5.0 (http://diana.imis.athena-innovation.gr/ DianaTools/index.php?r=tarbase/index) and miRTarBase (http://mirtarbase.mbc.nctu.edu.tw/). The symbols and names obtained were normalized using the official symbols and names from the National Center for Biotechnology Information (NCBI) database (http://www.ncbi.nlm.nih.gov/gene/). Different forms of TC were not classified during the data collection process. The majority of data collected referred to TGCT, which is the most common TC subtype.

Secondly, the current study obtained a human experimentally validated dataset of TFs and miRNAs from TransmiR (8), which collects and summarizes data from public literatures and biological experiments.

Thirdly, human TC host genes were extracted from miRBase (9) and the NCBI database, and each host gene was assigned an official symbol and identification code.

Fourthly, the differentially-expressed genes obtained in the present study were extracted from Cancer Genetics Web (http://www.cancerindex.org/geneweb/), NCBI, and relative literatures and papers. The differentially-expressed elements that were extracted included abnormally expressed proteins, genetic mutations, single nucleotide polymorphisms, and upregulated, downregulated, overexpressed and variable genes. The current study extracted TC-related genes from the GeneCards database (10) and relevant literature. TC-related genes affect the growth and migration of tumors, in addition to the efficacy of radial therapy and the clinical outcome of TC (11). Furthermore, popular TFs were obtained using the P-match method (12). All results were combined to produce a list of the TC relative genes. The present study only focused on the TFs obtained using TransmiR. The 1000-nucleotide (nt) promoter region sequences of the targets of differentially-expressed genes were downloaded from the University of California, Santa Cruz database (13). The $\mathrm{P}$-match method was used in combination with weight matrix and pattern matching approaches to identify transcription factor binding sites (TFBSs) in the 1000-nt promoter region sequences, and the TFBSs were subsequently mapped onto the promoter regions of targets. The P-match matrix library (http://www.gene-regulation.com/cgi-bin/pub/programs/ pmatch/bin/p-match.cgi), along with information collected from TRANSFAC ${ }^{\circledR}$ (http://www.gene-regulation.com/ pub/databases.html), contained sets of known TFBSs, and were used to search for a large number of different TFBSs. The vertebrate matrix and restricted high quality criterion of the matrix were used in particular for the search.

Finally, differentially-expressed miRNAs were extracted from miR2Disease (14) and relevant literatures. miR2Disease is a database that records miRNAs that are differentially-expressed in a number of human diseases. Novel experimental data was also obtained manually from recent studies and science citation index papers (http://apps.webofknowledge.com) regarding TC differentially-expressed miRNAs, using the key words 'seminoma', 'testicular germ cell tumor' and 'testicular cancer'.

Network construction. Data was organized into tables and subsequently used to form a graph. The TC-related factors, including TFs, genes and miRNAs, form the nodes, and the lines placed between these nodes signify the form of association between each element. As a result, the associations between elements form regulatory networks.

Three types of TC regulatory networks were formed, including the i) differentially-expressed network, ii) related network and iii) global network. Regulatory associations were extracted for TFs, miRNAs, and target and host genes from the dataset collected by the present study. This formed the global regulatory network, which combined all associations. Differentially-expressed elements were determined using the aforementioned fourth and final steps, and the associations between them, which were obtained from the global network, were selected to derive the differentially-expressed network. The related network was formed in a similar way.

\section{Results}

Differentially-expressed network of TC. Fig. 1 presents the regulatory associations of all differentially-expressed elements in TC. It is composed of TFs, miRNAs, and targets and hosts of miRNAs. This network was key for the latter half of the present study. The nodes in the network impact others directly or indirectly, and when combined, the associations reveal the primary cause of TC. If the genes and miRNAs are not differentially-expressed as presented in the network, then that individual is not expected to develop TC. Thus, TC may be prevented through the suppression of these differentially-expressed elements.

Similar to the host genes, all nodes are differentially-expressed in TC. The network contains three TFs: Tumor protein 53 (TP53), SMAD family member 4 (SMAD4) and phosphatase and tensin homolog (PTEN). The most significant pathways contain these three TFs. As presented in Fig. 1, TP53 and PTEN are together targeted by hsa-miR-222. TP53 is a common, mutated TF, which is differentially-expressed in various types of cancer (15), and the present study identified that it regulates hsa-miR-200c and hsa-miR-145 (Fig. 1). SMAD4 is a novel transcription factor, which is mutated in seminomatous GCTs (16), and the current study determined that it regulates hsa-miR-145 together with TP53. PTEN is an important junction of the network (17). PTEN and hsa-miR-21 form a self-adaptation association, forming the only circuit in the whole network (Fig. 1). The combined action of the nodes in the differentially-expressed network partially exposes the regulatory mechanisms of TC. The network indicates that it 


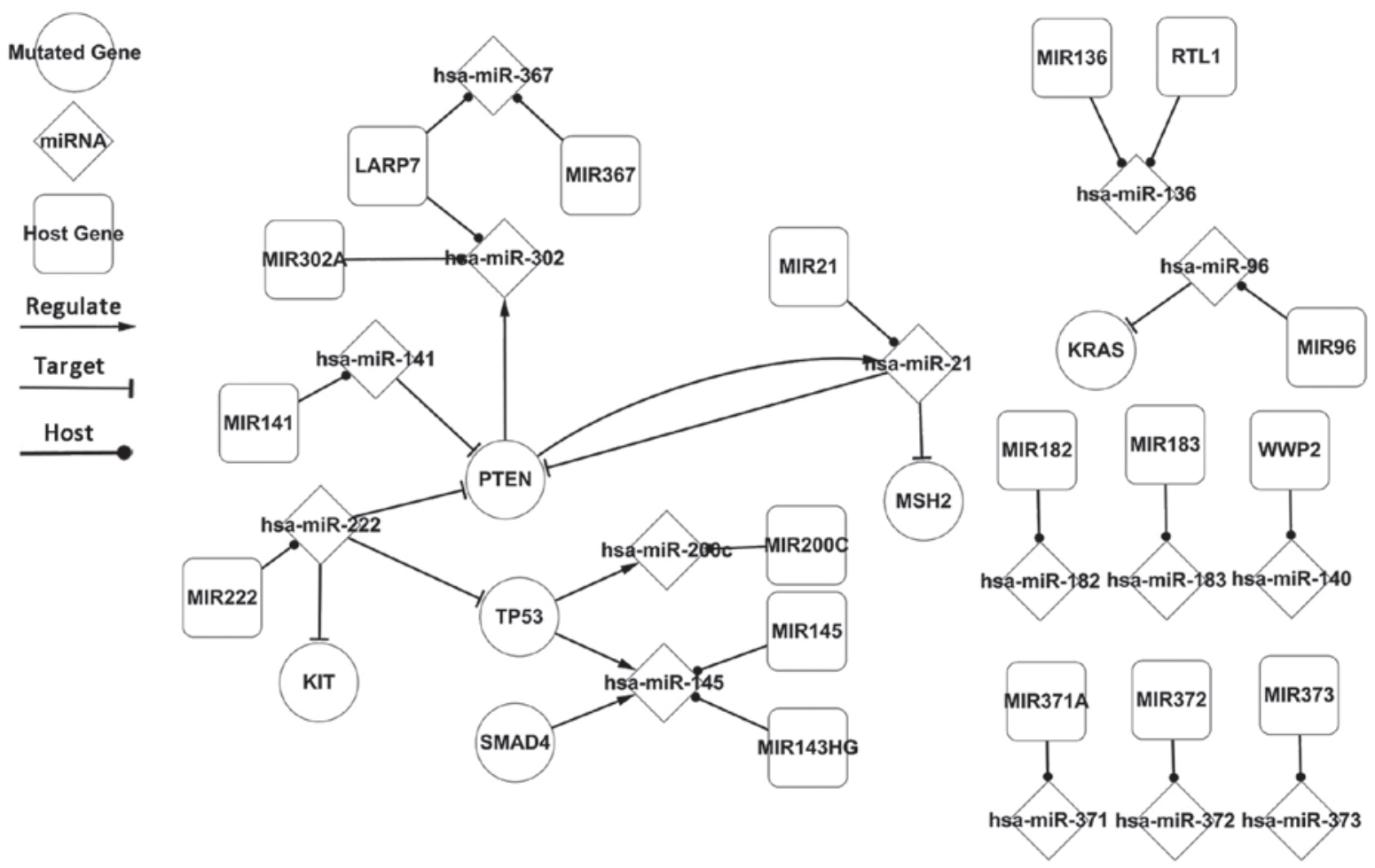

Figure 1. Differentially-expressed network of testicular cancer. miRNA/miR, microRNA.

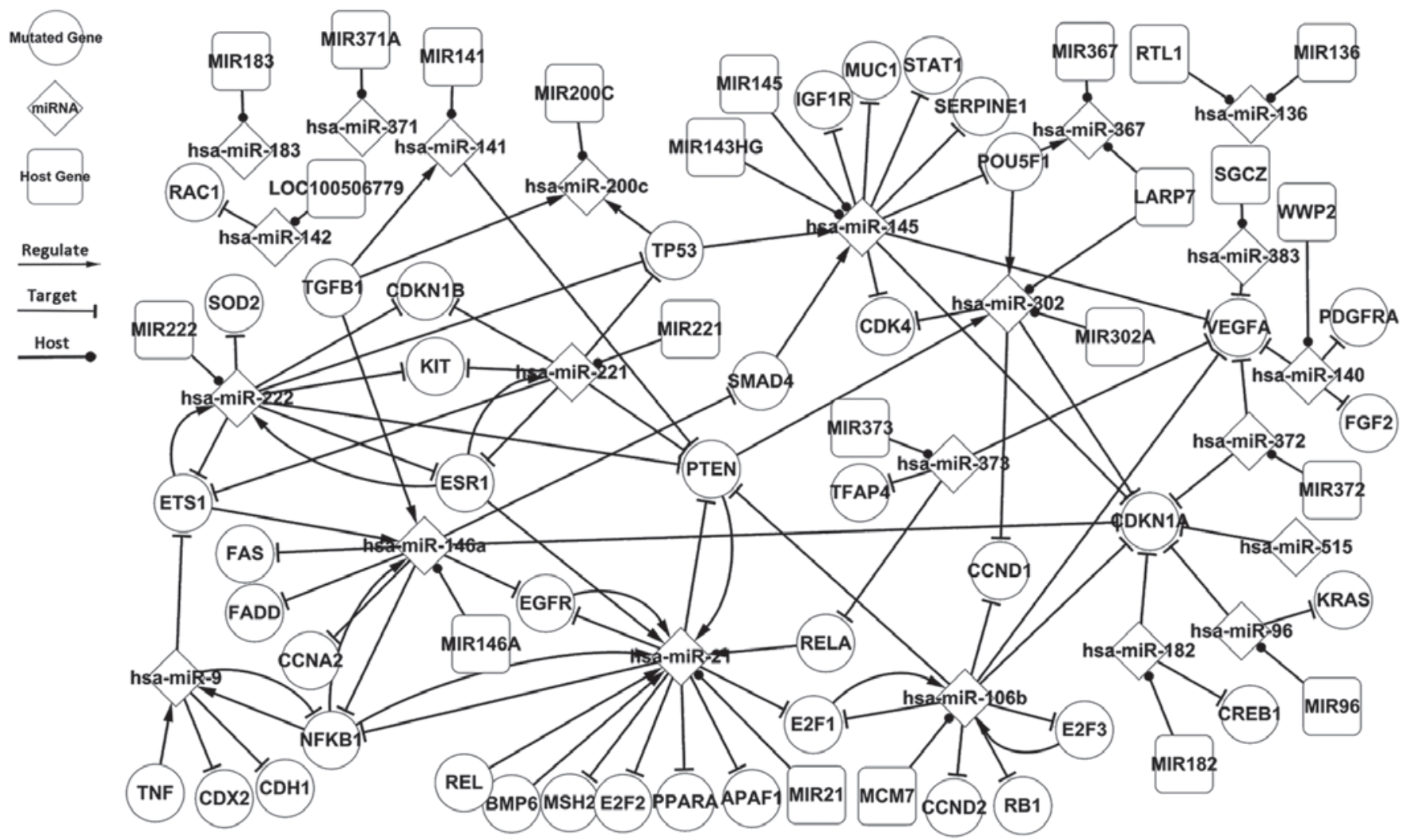

Figure 2. Related network of testicular cancer. miR, microRNA.

may be possible to artificially regulate differentially-expressed elements back to normal levels as a method to prevent or cure TC.
Related network of TC. Fig. 2 presents the mass regulatory associations between genes and miRNAs in TC. The related network includes the related elements collected by the present 
Table I. Upstream and downstream miRNAs of the PTEN gene in testicular cancer.

Location

Global network
Related network

Differentially-expressed

network
hsa-miR-106b, hsa-miR-141, hsa-miR-21, hsa-miR-221, hsa-miR-222

hsa-miR-21, hsa-miR-302
hsa-miR-141, hsa-miR-21

hsa-miR-222

$$
\begin{aligned}
& \text { hsa-miR-19a, hsa-miR-19b, } \\
& \text { hsa-miR-20a, hsa-miR-21, } \\
& \text { hsa-miR-214, hsa-miR-216a, } \\
& \text { hsa-miR-217, hsa-miR-221, } \\
& \text { hsa-miR-222, hsa-miR-26a, } \\
& \text { hsa-miR-494 }
\end{aligned}
$$

Downstream
hsa-miR-19a, hsa-miR-21, hsa-miR-21, hsa-miR-22, hsa-miR-25, hsa-miR-302a, hsa-miR-302b, hsa-miR-302c, hsa-miR-302d, hsa-miR-302f

PTEN

a

PTEN, phosphatase and tensin homolog; miR, microRNA.

study, in addition to predicted data. It is evident that the related network includes the differentially-expressed network. The related network of TC includes 18 TFs, 26 miRNAs and a number of targets. Fig. 2 presents certain integral, regulatory associations beyond the differentially-expressed network with a few additional pathways. The miRNA, hsa-miR-21, is regulated by 7 TFs and forms 3 self-adaptation associations. The TF, SMAD4, is targeted by hsa-miR-146a and regulates only 1 miRNA, hsa-miR-145. Other TF associations may also be identified within the related network, including TGFB1, which regulates 3 miRNAs, but is not targeted by other miRNAs, and ESR1, which regulates hsa-miR-21 and forms 2 self-adaptation associations with hsa-miR-221 and hsa-miR-222. The related network expands the additional topological associations to the pathogenesis of TC, and includes elements that are not differentially-expressed.

Global network of TC. The global network presents a greater number of comprehensive, regulatory associations compared with the differentially-expressed and related networks. The global network involves the whole experimentally validated network of the human body, and incorporates the related and differentially-expressed networks. All regulation elements and associations are common regulation situations that are present not only in TC, but in other diseases.

Host genes and miRNAs in TC. Host genes are not differentially-expressed in cancer, but are considered to be differentially-expressed if their miRNAs are. For example, la ribonucleoprotein domain family, member 7 hosts two differentially-expressed miRNAs, hsa-miR-367 and hsa-miR-302, which are each regulated by POU class 5 homeobox 1 (POU5F1). POU5F1 is targeted by hsa-miR-145, which is differentially-expressed, suggesting that the host genes may be useful in the regulatory pathway. Certain host genes contain a number of miRNAs that target genes or are regulated by other important TFs. The host genes and their differentially-expressed miRNAs may aid the understanding of TC pathogenesis.

Regulatory pathways of differentially-expressed genes. Differentially-expressed genes are presented in the differentially-expressed network. These genes directly regulate various differentially-expressed miRNAs, and therefore may be considered, to a certain extent, as a source of differential expression. Adjusting the differentially-expressed TFs to their normal condition may be a possible method for altering the genes associated with TC, via miRNAs, which would be affected the at the same time.

In order to clearly describe the features of the TC networks, the up- and downstream information of genes or miRNAs was extracted and presented in tables. Table I lists the successors and precursors of PTEN as determined by the related, differentially-expressed and global networks. PTEN is differentially-expressed itself and associated with other differentially-expressed genes or miRNAs in the network. PTEN and hsa-miR-21 form a self-adaptation association, thus affecting other genes as a unit. There are two miRNAs downstream of PTEN in the related network, which are each differentially-expressed. The expression of PTEN may be controlled as a source to adjust the differentially-expressed miRNAs that are regulated by PTEN. By controlling the expression of specific genes in the network, the abnormal expression of other elements may also be controlled, resulting in TC prevention.

Regulatory pathways of differentially-expressed miRNAs. Differentially-expressed miRNAs are important in the transcription network due to their association with other associated 
Table II. Upstream and downstream genes of hsa-miR-21 in testicular cancer.

Location

Global network
Differentially-expressed network
hsa-miR-21

Upstream

Downstream

\author{
EGFR, ESR1, ETV5, Foxo3a, \\ AP-1, NFIB, NFKB1, PTEN, \\ RAS/ERK, REL, RELA, REST, \\ BMP6, BMPR1a, BMPR1b, \\ STAT3, TCF4
APAF1, BASP1, BCL2, BMPR2, BTG2, CCR1, CDC25A, CDK2AP1, DAXX, DERL1, E2F1, E2F2, EGFR, EIF2S1, EIF4A2, ERBB2, FMOD, HNRNPK, ICAM1, IL1B, ISCU, JAG1, JMY, LRRFIP1, MARCKS, MEF2C, MSH2, MSH6, MTAP, MYC, NCAPG, NCOA3 PCBP1, PDCD4, PDHA2, PLAT, PLOD3, PPIF, PTEN, PTX3, RASGRP1, RECK, REST, RHOB, RPS7, RTN4, SERPINB5, SOX5, SPATS2L, SPRY2, TGFBI, TGFBR2, TGFBR3, TGIF1, TIAM1, TIMP3, TM9SF3, TNFAIP3, TOPORS, TP53BP2, TP63, TPM1, WFS1, WIBG

Related network

MIR21, BMP6, EGFR, ESR1, NFKB1, PTEN, REL, RELA

\author{
APAF1, E2F1, E2F2, EGFR \\ MSH2, NFKB1, PPARA, \\ PTEN
}

MIR21, PTEN

MSH2, PTEN

TFs in the related and differentially-expressed networks are represented in bold. miR, microRNA.

genetic elements, and therefore warrant further investigation. Theoretically, differentially-expressed miRNAs may be artificially regulated indirectly through regulating or targeting associations. Therefore, the present study extracted, compared and analyzed these miRNAs using the same method applied to analyze the differentially-expressed genes.

hsa-miR-21 is presented as an example in Table II. The data indicated that hsa-miR-21 is important in the transcription network. hsa-miR-21 is regulated by 7 genes, which are all TFs, and targets 7 genes, which include three TFs [epidermal growth factor receptor (EGFR), E2F transcription factor 1 (E2F1) and PTEN]. PTEN and hsa-miR-21 form an important self-adaptation association, which was discussed previously in the current study. Similarly, 2 self-adaptation associations were identified in the related network formed by the miRNA, hsa-miR-21, and 2 related TFs, EGFR and nuclear factor of $\kappa$ light polypeptide gene enhancer in B-cells 1 (NFKB1). hsa-miR-21 also indirectly affects other miRNAs, including hsa-miR-141, hsa-miR-106b and hsa-miR-222, by targeting the TFs that regulate these miRNAs.

As a further example, it was identified that hsa-miR-222 targets 2 TFs (PTEN and TP53), but is not regulated by any TFs in the differentially-expressed network. Two self-adaptation associations are derived in the regulated network by importing related elements. hsa-miR-222 and hsa-miR-21 have all three kinds of predecessors (upstream) and three types of successors (downstream) adjacent nodes. Other miRNAs belonging to this class include hsa-miR-96 and hsa-miR-141. These miRNAs are considered as the first class of miRNAs, as they have 6 adjacent nodes ( 3 successors and 3 predecessors) in the whole network.

Similarly to hsa-miR-302 and hsa-miR-145, other classes of miRNAs also have 5 adjacent nodes (2 successors and 3 predecessors). These miRNAs are considered the second class of miRNAs. The third class of miRNAs has 4 adjacent nodes (1 successor and 3 predecessors), and includes hsa-miR-367, hsa-miR200c and certain single miRNAs adjacent to the major nodes in the differentially-expressed network, such as hsa-miR-187 and hsa-miR-371. This information was derived through comparisons of Figs. 1 and 2.

Regulatory pathways of popular TFs. The P-match method was used to predict popular TFs in TC, in addition to the identification of additional pathways that may be involved in the process of differential expression. Fig. 3 presents the highlighted regulatory pathways, composed of miRNAs and popular TFs, which were derived from the related network. The related network primarily underlines important regulatory relationships beyond the differentially-expressed network. A total of 9 self-adaptation associations are presented in the 
Table III. Upstream and downstream miRNAs of the NFKB1 transcription factor in testicular cancer.

Location

Global network

Related network

Differentially-expressed network

$\begin{array}{ll}\text { NFKB1 } & \\ \text { Upstream } & \text { hsa-let-7a, hsa-miR-9, } \\ & \text { hsa-miR-146a, hsa-miR-146a, } \\ & \text { hsa-miR-15a, hsa-miR-21, } \\ & \text { hsa-miR-9, hsa-miR-146b-5p } \\ & \text { hsa-let-7a-3, hsa-let-7b, } \\ & \text { hsa-miR-10b, hsa-miR-125b, } \\ \text { hsa-miR-146a, hsa-miR-155, } & \text { hsa-miR-16, hsa-miR-17, } \\ & \text { hsa-miR-199a-2, hsa-miR-21, } \\ & \text { hsa-miR-214, hsa-miR-224, } \\ & \text { hsa-miR-29a, hsa-miR-29b, } \\ & \text { hsa-miR-29c, hsa-miR-34a, } \\ & \text { hsa-miR-365, hsa-miR-448, } \\ & \text { hsa-miR-9 }\end{array}$

NFKB1, nuclear factor of $\kappa$ light polypeptide gene enhancer in B-cells 1; miR, microRNA.

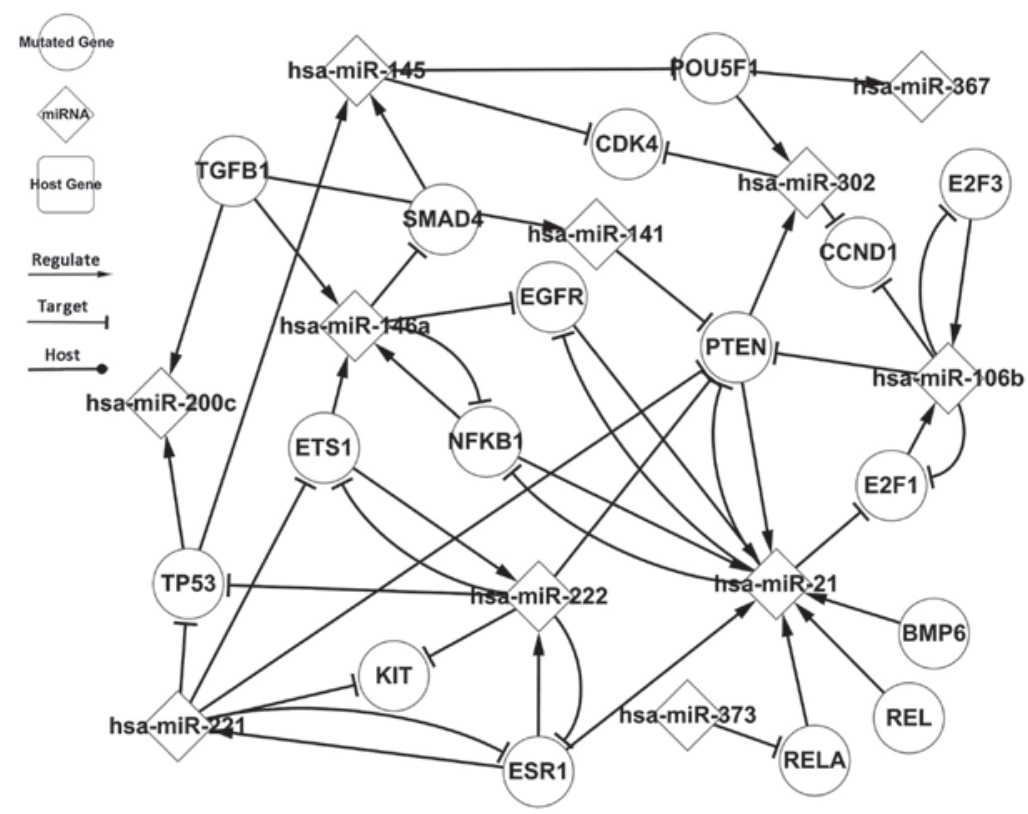

Figure 3. Highlighted regulatory pathways composed of miRNAs and popular transcription factors derived from the related network of testicular cancer. miRNA/miR, microRNA.

related network (Fig. 2), and involve the following TFs: PTEN, E2F1, E2F3, EGFR, ETS1, ESR1 and NFKB1, demonstrating that these TFs and miRNAs regulate one another.

The associations of NFKB1, a TF, were predicted using the P-match method, for which the up- and downstream information is presented in Table III. NFKB1 is involved in 3 self-adaptation associations in the related network, which includes hsa-miR-21, hsa-miR-146a and hsa-miR-9. Firstly, hsa-miR-21 is differentially-expressed. Secondly, whilst hsa-miR-146a is not differentially-expressed, it targets a
hsa-miR-9, hsa-miR-146a,

hsa-miR-146a, hsa-miR-21

hsa-miR-21

hsa-miR-9, hsa-miR-146a,

hsa-miR-146a, hsa-miR-21

hsa-miR-21 
3 miRNAs, including 2 differentially-expressed miRNAs (hsa-miR-141 and hsa-miR-200c). This indicates that TGFB1 may be located at the bottom level of the regulatory pathway. As an additonal example, POU5F1 is a gene that not differentially-expressed; however, all of the adjacent, interacting nodes of POU5F1 are differentially-expressed. Compared with the differentially-expressed network, the regulatory pathway links separated nodes together. The associated nodes further improve the regulatory pathway and the combined action of each node shows the regulatory progress and reveals pathology of TC.

\section{Discussion}

The present study derived three topological, regulatory networks composed of elements associated with TC. The key features of each pathway were discussed in detail, and regulatory pathways were highlighted, which included important elements involved in the regulatory process. Precursors and successors were listed for the major elements identified in the networks, and self-adaptation associations were identified. Furthermore, any particular nodes that warranted further research were indicated, and any predictions made using the P-match method may provide additional aspects of research.

Differentially-expressed elements and the associations between them are of particular importance, as it is suspected that these elements may be the primary cause of TC. If the relevant genes are differentially-expressed as described in the network, then it is expected that TC may develop. If these elements are able to be regulated back within normal ranges, then this may prevent the occurrence of TC.

The current study identified a number of key pathways involving differentially-expressed elements, which included three or more of these elements. For example, TP53 associates with 3 differentially-expressed miRNAs, regulating two of these (hsa-miR-200c and hsa-miR-145) and being targeted by one (hsa-miR-222). These pathways are critical in TC pathogenesis and illustrate a topological network of TC development. Certain pathways have not only been identified in TC, but also in other types of cancer. For example, the association whereby hsa-miR-21 targets PTEN also affects lung squamous cell carcinoma (18). Therefore, these associations between genes may be extended from one form of cancer to another.

In conclusion, the present study used the P-match method to predict TFs involved in TC pathogenesis, which suggested that potential associations may exist between differentially-expressed miRNAs and TFs. It is proposed that these differentially-expressed elements may be manipulated to achieve normal expression levels, subsequently preventing the development of TC. However, these associations remain to be experimentally validated in future studies, with the aim of eventually improving the prognosis, diagnosis and treatment of TC.

\section{Acknowledgements}

The present study was supported by grants from the National Natural Science Foundation of China (nos. 60973091 and 60905022) and the Natural Science Foundation of Jilin Province, China (no. 201330101166JC).

\section{References}

1. American Cancer Society. Testicular cancer. http://www.cancer.org/cancer/testicularcancer/index. Accessed January 15, 2015.

2. Eble JN, Sauter G, Epstein JI and Sesterhenn IA (eds): World Health Organization Classification of Tumours. Pathology and Genetics of Tumours of the Urinary System and Male Genital Organs. IARC Press, Lyon, 2004.

3. Hayes-Lattin B and Nichols CR: Testicular cancer: A prototypic tumor of young adults. Semin Oncol 36: 432-438, 2009.

4. Hobert O: Gene regulation by transcription factors and microRNAs. Science 319: 1785-1786, 2008.

5. Rodriguez A, Griffiths-Jones S, Ashurst JL and Bradley A: Identification of mammalian microRNA host genes and transcription units. Genome Res 14 (10A): 1902-1910, 2004.

6. Baskerville S and Bartel DP: Microarray profiling of microRNAs reveals frequent coexpression with neighboring miRNAs and host genes. RNA 11: 241-247, 2005

7. Cao G, Huang B, Liu Z, Zhang J, Xu H, Xia W, Li J, Li S, Chen L, Ding H, et al: Intronic miR-301 feedback regulates its host gene, ska2, in A549 cells by targeting MEOX2 to affect ERK/CREB pathways. Biochem Biophys Res Commun 396: 978-982, 2010

8. Wang J, Lu M, Qiu CU and Cui Q: TransmiR: A transcription factor-microRNA regulation database. Nucleic Acids Res 38 (Database issue): D119-D122, 2010.

9. Kozomara A and Griffiths-Jones S: miRBase: Integrating microRNA annotation and deep-sequencing data. Nucleic Acids Res 39 (Database issue): D152-D157, 2011.

10. Safran M, Dalah I, Alexander J, Rosen N, Iny Stein T, Shmoish M, Nativ N, Bahir I, Doniger T, Krug $\mathrm{H}$, et al: GeneCards Version 3: The human gene integrator. Database (Oxford) 2010: baq020, 2010.

11. Vladušić T, Hrašćan R, Krušlin B, Pećina-Šlaus N, Perica K, Bićanić A, Vrhovac I, Gamulin M and Franekić J: Histological groups of human postpubertal testicular germ cell tumours harbour different genetic alterations. Anticancer Res 34: 40054012, 2014

12. Chekmenev DS, Haid C and Kel AE: P-Match: Transcription factor binding site search by combining patterns and weight matrices. Nucleic Acids Res 33 (Web Server Issue): W432-W437, 2005.

13. Fujita PA, Rhead B, Zweig AS, Hinrichs AS, Karolchik D, Cline MS, Goldman M, Barber GP, Clawson H, Coelho A, et al: The UCSC Genome Browser database: Update 2011. Nucleic Acids Res 39 (Database Issue): D876-D882, 2011.

14. Jiang Q, Wang Y, Hao Y, Juan L, Teng M, Zhang X, Li M, Wang G and Liu Y: miR2Disease: A manually curated database for microRNA deregulation in human disease. Nucleic Acids Res 37 (Database Issue): D98-D104, 2009.

15. Petitjean A, Achatz MIW, Borresen-Dale AL, Hainaut $P$ and Olivier M: TP53 mutations in human cancers: Functional selection and impact on cancer prognosis and outcomes. Oncogene 26: 2157-2165, 2007.

16. Bouras M, Tabone E, Bertholon J, Sommer P, Bouvier R, Droz JP and Benahmed M: A novel SMAD4 gene mutation in seminoma germ cell tumors. Cancer Res 60: 922-928, 2000.

17. Di Vizio D, Cito L, Boccia A, Chieffi P, Insabato L, Pettinato G, Motti ML, Schepis F, D'Amico W, Fabiani F, et al: Loss of the tumor suppressor gene PTEN marks the transition from intratubular germ cell neoplasias (ITGCN) to invasive germ cell tumors. Oncogene 24: 1882-1894, 2005

18. Xu LF, Wu ZP, Chen Y, Zhu QS, Hamidi S and Navab R: MicroRNA-21 (miR-21) regulates cellular proliferation, invasion, migration and apoptosis by targeting PTEN, RECK and Bcl-2 in lung squamous carcinoma, Gejiu City, China. PLoS One 9: e103698, 2014. 